\title{
Erratum to: Motion of a rigidly rotating circle in terms of Minkowski geometry
}

\author{
Stanisław L. Bażański
}

Received: 20 October 2011 / Accepted: 28 October 2011 / Published online: 29 November 2011

(C) Springer Science+Business Media, LLC 2011

\section{Erratum to: Gen Relativ Gravit (2011) 43:3693-3703 DOI 10.1007/s10714-011-1254-y}

Unfortunately, the next to last paragraph on p. 3700 of the published version of this article contained mistakes. The complete corrected paragraph is printed below:

We start now with carrying out two geometric constructions which are needed to define the unit of measurement of angles in radians in the osculating two-plane $\pi\left(x^{1^{\prime}}, x^{2^{\prime}}\right)$ at a point $p\left(\varphi_{0}\right)$ of the trajectory $\mathcal{L}_{0}$. In the first, a chord of the osculating circle of $\mathcal{L}_{0}$ at $p\left(\varphi_{0}\right)$ is drawn in the direction parallel to the tangent vector $\ell$ at $p\left(\varphi_{0}\right)$. The chord intersects the osculating circle at two points, say $p_{1}{ }^{\prime}$ and $p_{2}{ }^{\prime}$, cutting off an arc of length $\mathcal{A}$, which subtends an angle $\Delta \psi$ at its centre $\mathcal{O}$. The second construction consists in drawing two two-dimensional timelike planes $\pi_{1}$ and $\pi_{2}$ which are Minkowski-orthogonal to the osculating two-plane at $p\left(\varphi_{0}\right)$ and pass along one of the segments $\left[\mathcal{O}, p_{1}^{\prime}\right]$ or $\left[\mathcal{O}, p_{2}^{\prime}\right]$ correspondingly. The planes intersect $\mathcal{L}_{0}$ at two points $p_{1}$ and $p_{2}$ which are the ends of an $\operatorname{arc} \mathcal{A}\left(p_{1}, p_{2}\right)$ of it, and the proper length of this arc is denoted by $\Delta \ell_{0}$.

The online version of the original article can be found under doi:10.1007/s10714-011-1254-y.

S. L. Bażański $(\bowtie)$

Institute of Theoretical Physics, University of Warsaw, Hoża 69, 00-681, Warsaw, Poland e-mail: bazanski@fuw.edu.pl 